\title{
Gouvernance environnementale dans les Alpes françaises. Le cas des stations moyennes
}

Jean-Christophe Dissart et Emmanuelle Marcelpoil

\section{(2) OpenEdition}

1 Journals

Édition électronique

URL : http://journals.openedition.org/tourisme/516

DOI : 10.4000/tourisme.516

ISSN : 2492-7503

Éditeur

Éditions touristiques européennes

Édition imprimée

Date de publication : 1 juin 2011

Pagination : 66-82

ISSN : 2109-5671

\section{Référence électronique}

Jean-Christophe Dissart et Emmanuelle Marcelpoil, « Gouvernance environnementale dans les Alpes françaises. Le cas des stations moyennes », Mondes du Tourisme [En ligne], 3 | 2011, mis en ligne le 30 septembre 2015, consulté le 19 avril 2019. URL : http://journals.openedition.org/tourisme/516 ; DOI : 10.4000/tourisme.516

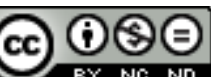

Mondes du tourisme est mis à disposition selon les termes de la licence Creative Commons Attribution - Pas d'Utilisation Commerciale - Pas de Modification 4.0 International. 


\section{Gouvernance environnementale dans}

\section{les Alpes françaises • Le cas des stations moyennes}

\section{JEAN-CHRISTOPHE DISSART}

[jean-christophe.dissart@cemagref.fr]

Chercheur, économie et aménagement du territoire

Cemagref, UR Développement des territoires montagnards

Résumé. L'objectif de cet article est d'analyser les processus de gouvernance environnementale dans le contexte de deux stations de sports d'hiver en phase de développement, situées dans le parc national de la Vanoise (France). L'analyse cible les unités touristiques nouvelles (UTN), autorisation de construire qui doit être déposée par les stations préalablement à tout projet de développement touristique significatif. Ainsi, les UTN sont des indicateurs pertinents des relations entre acteurs des projets, mais également du rôle de l'État, en évolution par rapport aux enjeux de développement touristique et de protection de l'environnement. À partir de documents écrits et d'entretiens avec des acteurs publics et privés, les résultats suggèrent une situation intermédiaire entre un modèle chaotique et régulé de gouvernance environnementale. De plus, le rôle des services de l'État est en évolution par rapport au ciblage de l'action, à l'expertise et à l'échelle géographique d'aménagement.

\author{
Emmanuelle Marcelpoll \\ [emmanuelle.george-marcelpoil@cemagref.fr] \\ Chercheur, économie et aménagement touristique \\ Cemagref, UR Développement des territoires montagnards
}

Abstract. The objective of this paper is to analyse environmental governance processes in the context of two expanding winter resorts located close to the Vanoise national park (France). The analysis focuses on New Tourism Units (NTUs), a building permit which must be filed by resorts prior to any significant tourism development. As such, NTUs are relevant indicators of the relations between project stakeholders and of the changing role of the State regarding tourism development and environmental protection issues. Based on written documents and interviews with both public and private stakeholders, results suggest an intermediate situation between a chaotic and a regulated model of environmental govemance. Moreover, the role of State services is changing with respect to focus of action, expertise and geographic scale of spatial planning. 
$\mathbf{E}$

n France, les stations de sports d'hiver représentent un secteur économique majeur, aujourd'hui confronté à plusieurs facteurs d'incertitude : maturité du marché du ski, forte concurrence entre stations, exigences croissantes de consommateurs versatiles (Afit, 2003), et injonction de la durabilité. S'agissant du développement durable, les stations en revendiquent les principes et multiplient les initiatives de certification, de labellisation, voire des démarches plus globales, à l'image de la récente charte de développement durable des stations, portée par l'Association nationale des maires de stations de montagne. Dans ce contexte, la présence de zones protégées à proximité des stations peut être considérée comme un avantage concurrentiel (notamment pour la saison estivale). L'objectif principal des stations en hiver reste néanmoins l'attraction et la fidélisation de la clientèle. À cet égard, les extensions et les interconnexions de domaines skiables constituent une dimension forte de l'amélioration de l'offre touristique. Cependant, ces projets suscitent souvent une opposition de la part des associations de protection de l'environnement. Ces dernières s'opposent à l'équipement de la montagne et aspirent à une approche plus équilibrée ${ }^{(1)}$ des pratiques actuelles.

Cette opposition entre développement touristique et protection de l'environnement n'est pas récente. Historiquement, elle a présidé à la définition des périmètres de protection et à la fonctionnalisation de l'espace (développement $v s$. protection), ce qui a déclenché plusieurs conflits violents (par exemple, dans le parc national de la Vanoise en 1969. 1971 (Mauz, 2002)). Cependant, les relations entre acteurs ont évolué au cours du temps. Les frontières entre opérateurs touristiques et acteurs de la protection de l'environnement ont pu s'estomper et des alliances entre services de l'État et monde associatif ont pu émerger. La force de telles alliances dépend des objectifs des projets, mais également de la vision du développement durable portée par les acteurs (Rivera et de Leon, 2004). Toutefois, créer et maintenir de telles relations impliquent que les acteurs concernés partagent un référentiel commun.

Ces différentes notions interpellent le concept de gouvernance, appliqué aux enjeux environnementaux. Ainsi, notre contribution $^{(2)}$ cherche à caractériser les processus de gouvernance environnementale dans les stations alpines. Une attention particulière est portée sur les évolutions du rôle des services de l'État, acteur majeur dans l'aménagement touristique en montagne. Les analyses apportées contribuent à une lecture des relations entre acteurs, eu égard à l'évolution touristique. La première partie présente le cadre d'analyse retenu en termes de gouvernance pour répondre aux questions posées. La deuxième partie revient sur les UTN comme descripteurs de ces processus de gouvernance environnementale, d'une part, et sur les caractéristiques des stations étudiées, d'autre part. Sur cette base, la troisième partie qualifie les processus de gouvernance environnementale rencontrés autour des procédures UTN en stations tandis que la quatrième partie évoque la question sensible de l'expertise de l'État et de son évolution. Enfin, la conclusion ouvre sur des perspectives de recherche à approfondir en termes de gouvernance environnementale en stations.

\section{CADRE D’ANALYSE}

La notion de gouvernance est largement mobilisée aujourd'hui, avec toutefois des objets et des finalités variés (Beritelli et al., 2007 ; Pechlaner, 1999). Ainsi, la gouvernance a été associée à la gouvernance des entreprises (corporate governance), à la gouvernance urbaine ou à la gouvernance à l'échelon mondial. La gouvernance, voire les gouvernances (Allemand, 2000) visent à décrire les mutations de l'action publique. En effet, les années 1990 ont été marquées par une complexité croissante, réelle ou perçue, de l'environnement économique, social et politique, par l'émergence de nouveaux acteurs sur la scène 
publique et par un enchevêtrement des niveaux décisionnels. Alors que la forme classique de gouvernement est remise en cause, le concept de gouvernance permet de reconnaître l'intensité croissante des processus de coordination entre acteurs. La gouvernance se présente alors comme l'ensemble des arrangements formels et informels entre acteurs, à partir desquels les décisions sont prises et mises en œuvre (Le Galès, 1995, pp. 57-95). Au-delà de la pluralité d'acteurs, la gouvernance rend compte de la possible émergence d'un acteur collectif (Gerbaux et Marcelpoil, 2006).

Le domaine environnemental n'a pas échappé pas à la reconfiguration de l'action publique et à la diffusion de la notion de gouvernance. Il a d'ailleurs pu jouer un rôle précurseur. Par la complexité des questions soulevées (externalités, usages de ressources collectives), par la pluralité des acteurs et des échelles (Flagestad et Hope, 200 I), l'environnement peut être perçu comme un laboratoire de nouvelles gouvernances (Clivaz et al., 2004 ; Svensson et al., 2005 ; Theys, 2003). La mobilisation de la notion de gouvernance dans le champ environnemental met en exergue les dimensions temporelle et spatiale (Beaurain, 2004). En particulier, Beaurain (ibid.) rappelle que "les contraintes posées par le respect de l'environnement apparaissent à la fois comme des problèmes à résoudre à court terme tout en engageant le temps long de l'intergénérationnel". Dès lors, la coordination entre acteurs, situés géographiquement et inscrits dans une période plus ou moins longue, est avancée comme une voie de résolution de la prise en compte des contraintes environnementales. Ces processus de coordination d'acteurs, qualifiés de gouvernance environnementale, supposent également des rapprochements entre les perceptions des parties prenantes et, in fine, le dépassement des seuls outils économiques, à l'image des analyses coûts-avantages. Ces modalités d'émergence de nouvelles coordinations d'acteurs, en renvoyant aux représentations de l'environnement portées par les divers groupes d'acteurs, avancent l'importance du territoire dans lequel les enjeux environnementaux sont localisés. Nous rejoignons ici l'idée que la perception de l'environnement est à associer aux conditions de développement du territoire, à la perception que véhiculent les acteurs tant économiques que ceux relevant de la sphère environnementale, les acteurs tant privés que publics.

En définitive, au-delà de conflits souvent virulents, en relation avec l'affirmation de la société civile, les enjeux environnementaux peuvent également rapprocher des acteurs que tout oppose en apparence ou faire émerger des alliances pour une action collective. Apparaît alors un processus de gouvernance environnementale, décrit par Theys (2003) comme des étapes successives par lesquelles des acteurs nombreux, qui, de prime abord, ne partagent ni le même intérêt ni la même échelle d'action, vont progressivement construire une représentation partagée du problème auquel ils sont confrontés, se fixer des objectifs et retenir des solutions qui seront mises en œuvre collectivement, sans que cette représentation, ces objectifs ou cette interaction soient déterminés à l'avance.

Notre lecture des processus de gouvernance environnementale mobilise le modèle de Theys (2003) appliqué à des exemples de dossiers d'unités touristiques nouvelles (UTN). Les UTN constituent des descripteurs pertinents de la relation entre développement touristique et protection de l'environnement. Elles obligent à analyser la perception de l'environnement mise en avant par les différentes parties prenantes, acteurs économiques comme acteurs relevant de la sphère environnementale. Les UTN peuvent donc être perçues comme des supports de lecture de la gouvernance environnementale.

Cependant, l'affirmation de la notion de gouvernance dans le champ environnemental ne résout pas tous les enjeux. L'affirmation de la multiplicité des acteurs, de la démarche dite participative questionne la responsabilité avec une dilution possible de cette der- 
nière. De même, la place des nouvelles formes participatives dans le processus décisionnel est souvent pointée du doigt avec la complexité de la démocratie. Autant de limites qui peuvent déboucher sur des décisions inutiles, voire trop tardives.

Dans ce contexte, Theys (2003) a visé une opérationnalisation de la notion de gouvernance environnementale grâce à une décomposition en quatre dimensions (cf. tableau 1). De fait, la gouvernance s'apparente plutôt à un labyrinthe complexe de principes, outils, institutions et procédures dont la cohérence n'est pas d'emblée évidente. Au-delà de cette confusion apparente, Theys détaille quatre composantes du concept de gouvernance environnementale : un souci évident de relégitimation et de modernisation de l'action publique appelant à plus de transparence; des formes originales et multiples de coordination non hiérarchiques des actions collectives (contrats, outils basés sur le marché) ; des formes alternatives de rationalité (de la rationalité instrumentale à court terme au principe de précaution); enfin, un transfert de pouvoir vers la société civile, les collectivités locales ou des institutions autonomes. Le tableau 1 détaille ces quatre dimensions, lesquelles fournissent un cadre d'analyse pour les études de cas, ainsi qu'une référence normative en vue de positionner le processus étudié. Même

\section{Tableau I - Les quatre dimensions de la gouvernance environnementale}

\section{Moderniser l'action publique, \\ en accroître la légitimité et la crédibilité}

(gérer la confiance et l'acceptabilité)

- Réforme du secteur public

- Transparence

- Évaluation, contrôle, accountability

- Autorités indépendantes

- Séparation régulateur/opérateur

- Consultations et débats publics ;

démocratisation des procédures

- Mise en œuvre plus efficace (enforcement)

\section{3. Étendre la rationalité réflexive} ou procédurale

(gérer l'incertitude et la complexité)

- Principe de précaution

- Développement durable

- Évaluation des risques, études d'impact, réflexivité

- Calcul économique et réformes comptables

- Accès à l'information, transparence, traçabilité, indicateurs, audits...

- Conférences de consensus

- Pluralité de l'expertise, autorités indépendantes

- Science "post-normale"

- Déontologie et comités d'éthique

\section{Développer des mécanismes}

non autoritaires de coordination et de régulation de l'action collective

(gérer la pluralité et la mobilisation)

- Extension du contrat

- Partenariat public-privé

- Incitations économiques (permis négociables, taxes) ; compensations

- Accords volontaires

- Conventions et protocoles flexibles ("accords cadres")

- Politiques constitutives

- Autorités régulatrices

- Mécanismes de médiation

- Intégration et transversalisation

- Mise en œuvre négociée

- Réseaux informels

\section{Changer le pouvoir}

(gérer les rapports de force)

- Transferts de souveraineté

(aux institutions supranationales)

- Décentralisation

- Subsidiarité active

- Droits de propriété

- Normalisation volontaire

(exigences essentielles, autocertification)

- Délégation au secteur privé ou aux ONG

- "Autogestion" des biens publics par

des communautés d'usagers

- Institutions de mutualisation si, selon Theys (2003), la gouvernance ne devrait pas être considérée comme une "boîte à outils managériale”, ces critères d'ana- lyse peuvent être utilisés pour opérationnaliser le concept de gouvernance environnementale et faciliter son utilisation empirique. 


\section{LES UTN COMME RÉVÉLATEURS DE PROCESSUS DE GOUVERNANCE}

\section{La procédure UTN}

Sur cette base, les UTN sont retenues comme descripteurs des processus de gouvernance environnementale. La pertinence de ce choix s'inscrit dans l'histoire du développement des stations d'hiver en France. La création et l'expansion des stations ont constitué une solution à l'exode rural et au manque d'emplois (Pialat, 1970). En pratique, deux modes principaux d'implantation de stations peuvent être distingués. Dans un premier temps, les stations pionnières, créées au début du $\mathrm{XX}^{\mathrm{e}}$ siècle, étaient structurées autour des villages existants et accueillaient des activités touristiques estivales. Ces projets ont impliqué des acteurs extérieurs mais ont également bénéficié d'une participation significative d'acteurs locaux, publics et privés. Dans un second temps, les stations ex nibilo, voulues et portées par l'État (Plan neige), ont permis de développer une véritable industrie touristique en montagne, calquée sur le modèle urbain. Ce type de station a suscité de vives critiques environnementales dès les années 1970 ; certains qualifiant même la neige d'empoisonnée (Arnaud, 1975). Dès lors, en 1977, une directive nationale d'aménagement de la montagne a affirmé le caractère exceptionnel de la montagne et les précautions à prendre en termes d'urbanisation et d'équipement en montagne.

Dans la foulée, l'outil UTN est créé en 1979 (par décret) afin d'encadrer l'urbanisation de la montagne, via les études d'impact. Les opérateurs touristiques doivent, par l'intermédiaire des collectivités locales concernées, déposer un dossier d'UTN pour leur projet. En ce sens, les UTN sont un baromètre des relations entre l'État et les pouvoirs locaux (Péry, 1995), mais également un descripteur des stratégies suivies à la fois par les gestionnaires des espaces protégés et les promoteurs du développement touristique $^{(3)}$.

La procédure UTN a connu des évolutions majeures, d'abord en 1985, puis en 2006, à la fois en termes de critères d'équipement, d'échelle géographique de dépôt des demandes et des acteurs impliqués. À la suite de la décentralisation de 1982-83, le dispositif a été modifié par la loi Montagne de 1985. Cette dernière a notamment instauré la construction en continuité des équipements existants ainsi que diverses modifications dans le dépôt de dossiers UTN.

À titre d'exemple, le nombre d'études à fournir lors de la procédure a été augmenté à cinq, avec diagnostic (état du site), caractéristiques du projet, risques naturels, impacts sur l'environnement, et conditions d'équilibres économique et financier (Vlès, 2006).
Néanmoins, au cours du temps, les UTN sont devenues de médiocres outils pour traiter les extensions limitées de domaine skiable, la restructuration des stations et les interconnexions croissantes de domaine skiable (Pérès, 2007). Le décret UTN de 2006 a alors introduit deux changements majeurs dans la procédure d'autorisation : un ajout de trois volets aux dossiers UTN (historique de l'enneigement local ; ressource et qualité de l'eau ; exploitation et promotion des hébergements et équipements) et une instruction des dossiers désormais réalisée à deux échelles géographiques (département ou massif) suivant la nature (remontée mécanique, hébergement ou équipement touristique) et la taille du projet, renforçant ainsi le rôle des acteurs locaux, à différentes échelles.

Les critères des dossiers UTN soulignent donc le rôle accru des acteurs locaux mais également l'existence de lacunes dans la procédure d'autorisation ( $c f$. tableau 2). En dépit de son objectif affiché de gérer la croissance, le décret révisé ne peut empêcher une commune d'entreprendre plusieurs projets d'extension du domaine skiable, tant que chaque extension fait moins de 10 hectares. La procédure d'autorisation souligne aussi le rôle majeur du préfet (au niveau du département ou du massif) et de la commune, avec les services de l'État dans un rôle intermédiaire et principalement de consultation. 
Selon Frottier (2007), le décret UTN de 2006 devrait avoir plusieurs conséquences : une interaction accrue avec décideurs et acteurs du développement touristique ; une prise en compte marquée de la spécificité locale par la commission chargée du projet et un rapprochement accru entre décideurs et gestionnaires de projet. En mettant l'accent sur le système d'acteurs, le décret de 2006 entérine les UTN comme indicateurs des stratégies d'acteurs et comme descripteurs d'une gouvernance environnementale.

\section{Le cas d'étude : deux stations de sports d'hiver savoyardes}

Les données empiriques de notre contribution reposent sur des dossiers UTN relatifs à des stations de sports d'hiver localisées en Savoie. Malgré l'absence de suivi exhaustif des permis UTN, il est généralement admis que la décennie 1980-1990 a connu des projets UTN d'ampleur (en termes tant quantitatifs que qualitatifs) dans les stations d'altitude renommées. Les années 2000 présentent davantage de projets relatifs aux retenues collinaires (pour des projets de neige de culture) et au développement de stations de taille moyenne.

Deux stations de taille moyenne ont été ici retenues à dire d'experts $^{(4)}$ : Sainte-Foy-Tarentaise et Val Cenis-Termignon. Ces deux sites sont emblématiques des stations dites moyennes ou stations de moyenne montagne. Ces der-

\section{Tableau 2 • Critères pour la procédure UTN}

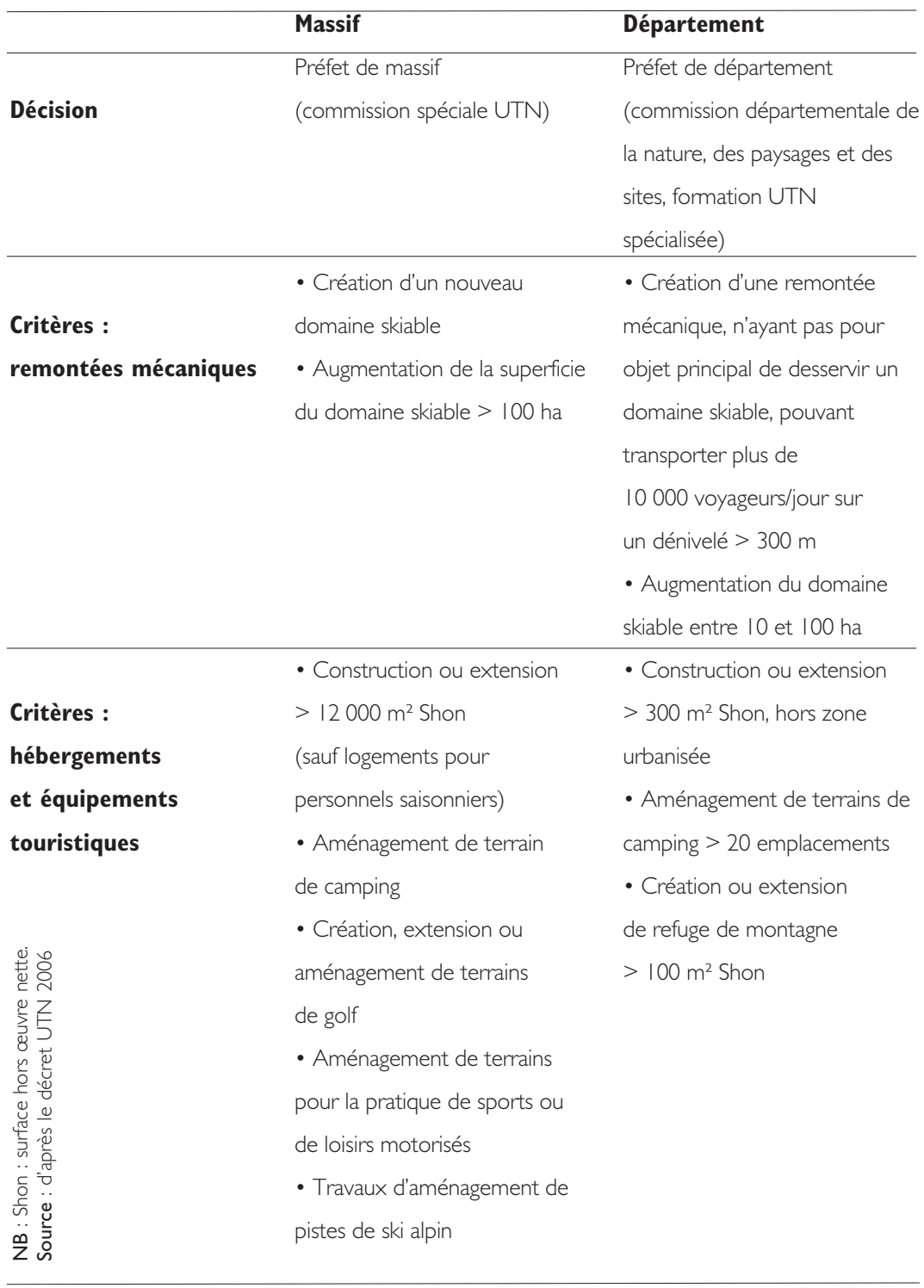

nières sont associées au qualificatif, souvent péjoratif, de moyen (Marcelpoil, François, 20I0) : stations moyennes en étendue de domaine skiable, en taille de parc d'hébergement touristique, en altitude, en chiffre d'affaires... Plus largement, il n'existe pas de définition officielle des stations moyennes et le recours à la typologie en générations (Cumin, 1970) ne permet pas de les discriminer, car elles relèvent de différentes générations.

Les dossiers UTN de ces sites illustrent la situation de stations moyennes dont l'objectif essentiel vise à développer des infrastructures touristiques (remontées mécaniques, 
Figure | - Localisation des stations de Sainte-Foy-Tarentaise et Val Cenis-Termignon (Savoie)

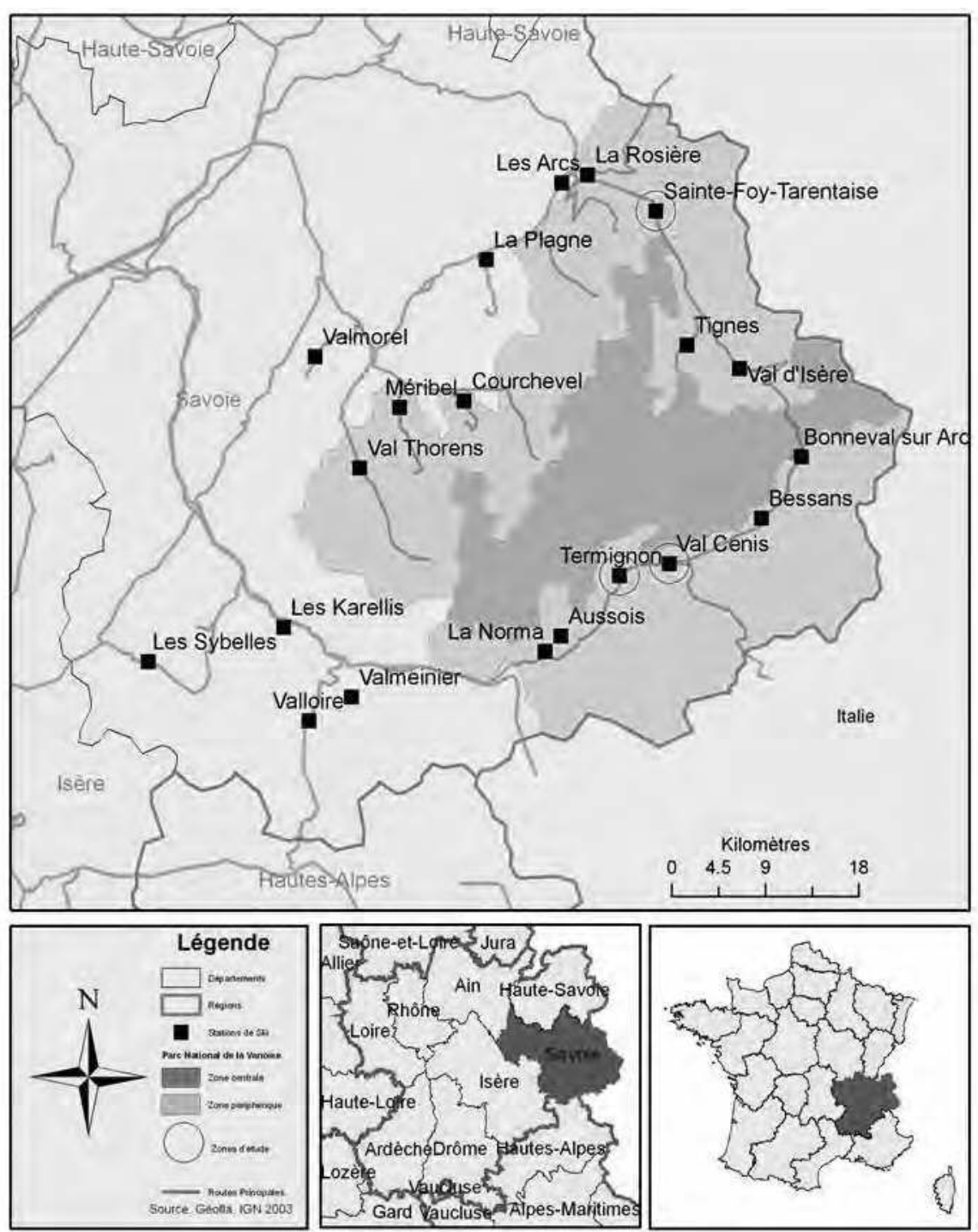

hébergement touristique) à l'image de leurs consœurs d'altitude. Enfin, dernier élément de choix : les procédures UTN de ces deux sites, situées en zone périphérique du parc national de la Vanoise, sont confrontées à de forts enjeux environnementaux et leur actualité nous permet de saisir les alliances entre acteurs et les phénomènes de gouvernance environnementale.

En pratique, les données mobilisées sont essentiellement de deux types : des documents factuels sur les dossiers UTN (rapports des services de l'État, textes réglemen- taires) et des entretiens semi-directifs auprès d'acteurs variés. Les personnes interrogées ont été sélectionnées en fonction de leur implication dans le dossier UTN et de leur perception du projet. En tant que tel, le groupe de répondants comprenait agents de l'État et des collectivités territoriales, élus (y compris membres de l'opposition politique), associations de protection de l'environnement, opérateurs de remontées mécaniques, gestionnaires de parc et promoteurs immobiliers. Une trentaine d'entretiens ont été menés sur les deux sites. Certains acteurs ont pu être interrogés sur les deux cas d'étude. Le guide d'entretien comprenait des sections relatives à l'histoire du projet, à la perception des enjeux, des impacts du projet et des relations entre acteurs et, plus largement, au débat entre équipement et protection.

Sur la base de ce matériau empirique et en suivant Yin (2009) quant à la nécessité de croiser les informations disponibles, nos méthodes d'analyse ont consisté à synthétiser les données issues de sources multiples, à réduire les biais potentiels et à renforcer l'interprétation des informations disponibles. Un guide d'interprétation fondé sur les quatre dimensions de la gouvernance environnementale (Theys, 2003) a également été utilisé. En particulier, les documents et entretiens ont été spécifiquement analysés à l'aune des indicateurs listés dans le tableau 1. 


\section{LES UTN}

EN STATIONS MOYENNES : QUELS PROCESSUS

\section{DE GOUVERNANCE ?}

Cette partie présente les caractéristiques essentielles des UTN des stations étudiées, ainsi que les éléments de gouvernance environnementale.

Les stations de Val Cenis et de Termignon, en bordure du parc national de la Vanoise (cf. figure 1), sont localisées dans la vallée de la Maurienne, territoire longtemps marqué par l'activité industrielle. L'UTN concerne l'interconnexion des domaines skiables de Val Cenis (communes de Lanslebourg et Lanslevillard) et de Termignon (communes de Termignon et Sollières-Sardières); la procédure d'autorisation a débuté en 20042005.

La station de Sainte-FoyTarentaise (dénommée Sainte-Foy), quant à elle, est située dans la vallée de la Tarentaise où le tourisme est une activité majeure - la vallée accueille les plus grandes stations françaises. Sainte-Foy entre dans la deuxième phase de son développement et a sollicité une autorisation UTN en 2004.

Tel qu'illustrés ( $c f$. tableau 3) par le parc d'hébergement (inférieur à 20000 lits), par le nombre de remontées mécaniques et par la taille du domaine skiable (inférieur à $100 \mathrm{~km}$ de pistes), Val CenisTermignon et Sainte-Foy sont des stations de taille moyenne. De plus, leur domaine skiable débute à $1500 \mathrm{~m}$ au plus, une altitude considérée comme critique face aux effets attendus du changement climatique.

Dans les années 1960, l'intervention de l'État et le Plan neige ont contribué à la création de stations importantes caractérisées par la planification urbaine, le contrôle du foncier et la rationalisation économique.

C'est dans ce contexte que la station de Val Cenis a été créée en 1967, à l'initiative des communes de Lanslebourg et de Lanslevillard. Grâce à une expansion maîtrisée, sa croissance, durant les années 1970-80, a permis de concilier équipement et protection. Son plan pluriannuel de développement touristique (PPDT) a été validé en 1981 et constitue le cadre de référence pour la restructuration actuelle du domaine skiable. La station de Termignon a été créée au début des années 1980. Son PPDT, validé en 1985, prévoyait l'équipement de 1500 lits (Zac de $14500 \mathrm{~m}^{2}$ de Shon), deux télésièges et quatre téléskis. Les services techniques de l'État ont, dès l'origine, souligné le potentiel limité du site (espace restreint, qualité environnementale élevée). Au fil des ans, le déficit récurrent des remontées mécaniques a confirmé les inquiétudes des services techniques, bien que la redevance liée à la production hydroélectrique ait longtemps pu le combler.

Tableau 3 • Chiffres clés des stations de Val Cenis, Termignon et Sainte-Foy (2004-2005)

\begin{tabular}{llll}
\hline & Val Cenis & Termignon & Sainte-Foy \\
\hline Lits (nombre) & 10000 & 2000 & 3000 \\
& $($ dont $83 \%$ lits marchands) & & \\
\hline Altitude du domaine skiable & $\mid 400-2800 \mathrm{~m}$ & $1300-2465 \mathrm{~m}$ & $1520-2620 \mathrm{~m}$ \\
\hline Remontées mécaniques & 2 télécabines $; 10$ télésièges; & 2 télésièges $; 4$ téléskis & 4 télésièges \\
\hline Pistes & 7 téléskis & & $13^{(1)} ; 25 \mathrm{~km} ; 62$ ha \\
(nombre ; longueur ; superficie) & $43 ; 80 \mathrm{~km} ; 250$ ha & & $15^{(2)} ; 30 \mathrm{~km} ;$ \\
\hline
\end{tabular}

Note : (I) ski facile surtout; (2) ski plutôt difficile.

Sources : Asadac (2004) ; DCSA, Kalysteo, Comète, SIVC (2005), offices du tourisme. 
Les années 1990 ont été synonymes de difficultés majeures pour les stations de taille et d'altitude moyennes : manque de neige, mutation du marché des sports d'hiver, effondrement du marché immobilier. Val Cenis, touchée par les difficultés financières, s'est orientée vers deux solutions : un projet de connexion avec l'Italie pour attirer la clientèle de la métropole turinoise, projet rejeté pour des raisons à la fois techniques et environnementales ; une augmentation de la capacité d'hébergement via de nouveaux lits marchands. En 2001-2002, Lanslebourg s'est vu autoriser la construction de $40000 \mathrm{~m}^{2}$ de Shon (équivalente à 3500 lits), dont la première livraison a eu lieu en 2003-04 (en partie stimulée par des avantages fiscaux liés au zonage en revitalisation rurale).

Aussi, alors qu'elle n'avait plus investi dans les remontées mécaniques depuis une quinzaine d'années, la station a lancé un programme de restructuration de son domaine skiable (un investissement de 20 millions d'euros entre 2000 et 2005). Ce domaine skiable était perçu comme un facteur limitant (en termes de taille et de qualité) pour commercialiser les nouveaux lits. Le cercle vicieux hébergement-domaine skiable, où la mise en marché de nouveaux lits est rentabilisée par l'extension du domaine skiable (et inversement), était alors enclenché.

Début 2003, les communes de
Termignon et de Sollières-Sardières ont décidé d'étudier l'aménagement du domaine skiable de la Turra et la liaison entre les stations de Val Cenis et de Termignon. L'extension demandée sur le massif de la Turra est située en site vierge avec de forts enjeux environnementaux (en particulier dans la combe de Cléry), entraînant une forte opposition de la part des associations écologistes. Le rappel des dates clefs montre la virulence des débats :

- 24 mars 2005. Avis favorable de la commission spécialisée UTN ;

- 22 avril 2005. Rejet du dossier par le préfet de massif (pour raison d'impacts environnementaux défavorables) ;

- 13 mai 2005. Appel des maires ; - 26 juillet 2005. Confirmation $\mathrm{du}$ rejet par le ministère de l'Équipement, établissement de la liste des remontées mécaniques envisageables et exclusion de tout aménagement dans la combe de Cléry ;

- août 2005. Dépôt d'un nouveau dossier, qui maintient un projet d'aménagement dans la combe de Cléry ;

- 29 mai 2006. Autorisation du projet sous conditions : prise d'un arrêté de protection du biotope dans le vallon de Cléry préalable à tout équipement de ce secteur ; attention particulière aux travaux de construction ; constitution d'une commission d'accompagnement et de suivi ;
- 27 juillet 2006. Recours juridique déposé par des associations de protection de l'environnement ; - 26 mars 2009. Rejet de l'appel.

À l'instar de Termignon, la station de Sainte-Foy a été créée tardivement, au début des années 1980, avec une gestion par régie directe. En raison de difficultés financières attendues et de la qualité environnementale du site, son PPDT a été validé en 1984, avec un accord "de principe" assorti de plusieurs conditions. Le projet comprenait un plan d'urbanisation de $85000 \mathrm{~m}^{2}$ ainsi qu'un domaine skiable avec 11 remontées mécaniques, principalement localisées sur le versant nord-ouest de la Foglietta et, secondairement, sur son versant sud, vers le vallon du Clou. En 1990, trois premiers télésièges ont été construits sur le versant nord-ouest de la Foglietta, desservant la moitié du domaine skiable autorisé. La station ouvrit au début de la saison hivernale 1990-1991, au moment des premiers hivers sans neige.

À Sainte-Foy, les projets immobiliers autorisés en 1988 n'ont pas pu démarrer en raison du manque d'investisseurs. Les projets furent même annulés en 1991, parce que la procédure d'autorisation UTN n'avait pas pris en compte l'avis du public et que la commission UTN n'avait pas été consultée. Un nouveau dossier UTN a été déposé en 1992 pour confirmer le cadre légal du développement de la station et pour renouveler les auto- 
risations initialement obtenues en 1988. Le dossier UTN reconnaissait le contexte (alors morne) du marché immobilier en mettant la priorité sur des programmes de petits lots (chalets). L'autorisation arriva la même année, avec à nouveau plusieurs conditions, dont un calendrier pour les opérations de construction et un contrôle du risque d'endettement excessif. Une urbanisation de $30000 \mathrm{~m}^{2}$ et trois remontées mécaniques ont tout d'abord été autorisées. La poursuite de l'équipement était soumise à une nouvelle demande d'autorisation UTN et à une évaluation de la première phase du programme de développement.

De manière générale, et contrairement à Val Cenis-Termignon, la procédure d'autorisation UTN s'est déroulée plutôt aisément à Sainte-Foy, compte tenu notamment d'enjeux environnementaux moins sensibles. En effet, la demande d'UTN de Sainte-Foy faisait partie d'un programme de développement progressif de la station (deuxième phase). En 2004, la commune a demandé l'autorisation de construire $40000 \mathrm{~m}^{2}$ d'hébergement supplémentaires sur le site de Bon Conseil, ainsi qu'un télésiège et ses pistes associées. L'équipement supplémentaire doit faire partie d'une zone d'aménagement concerté (Zac) concédée à la Société d'aménagement de la Savoie (un partenariat public-privé) et respecter une charte architecturale qui impose un bâti traditionnel (bois, murs extérieurs en pierre, toits de lauze). Les hébergements sont prévus sous forme de résidences de tourisme ou de formule locative à gestion hôtelière et réalisés par des promoteurs déjà connus à Sainte-Foy. À l'issue de la deuxième phase de développement, prévue en 20112012, la capacité d'hébergement de la Zac serait portée à 4650 lits.

Ce projet UTN a été autorisé en janvier 2005 par le préfet, assorti de contraintes : réaliser un schéma fonctionnel traitant des équipements nécessaires aux activités touristiques ; mettre au point un plan urbain et architectural intégrant les enjeux relatifs aux espaces publics ; augmenter la capacité d'accueil (via des lits marchands supplémentaires) avec des accords pour garantir la banalisation de ces nouveaux lits; prendre en compte les besoins en logement des saisonniers ; assurer l'adéquation entre construction supplémentaire et adduction d'eau et traitement des effluents ; réaliser une évaluation fonctionnelle et financière de la station à mi-parcours et au terme de la deuxième phase ; mettre en place un comité de contrôle de ces contraintes.

Toutefois, cette deuxième phase du développement de Sainte-Foy a évité la difficile question de l'aménagement du vallon du Clou, un espace situé sur le versant sud de la Foglietta, limité en superficie mais important du point de vue environnemental. Un tel aména- gement pourrait être envisagé par la commune pour une troisième phase du programme de développement, avec certainement de fortes dissensions entre les acteurs du projet.

La gouvernance environnementale dans les stations de Val CenisTermignon et de Sainte-Foy peut être analysée à partir des forces et faiblesses observées pour chacune des dimensions détaillées par Theys (2003). À partir des documents écrits et des entretiens semidirectifs relatifs aux cas étudiés, il est possible de suggérer une typologie des modèles de gouvernance environnementale des stations de sports d'hiver (cf. tableau 4).

L'analyse montre que les processus de gouvernance environnementale à l'œuvre dans les deux projets UTN sont situés entre un modèle "chaotique" et un modèle "régulé". Les processus de gouvernance environnementale peuvent être identifiés le long d'un gradient de modèles structurés autour de quatre dimensions fondamentales de la gouvernance environnementale, sur lesquelles les deux cas analysés diffèrent.

Ainsi, le projet de Sainte-Foy montre des éléments d'un modèle plus "régulé", alors que celui de Val Cenis-Termignon tend davantage vers un modèle "chaotique". La procédure d'autorisation qui s'est déroulée à Sainte-Foy était à la fois plus prévisible et plus sereine, avec des acteurs engagés dans un dialogue sincère sur les 


\section{Tableau 4 - Modèles de gouvernance environnementale Application à Val Cenis-Termignon et Sainte-Foy-Tarentaise}

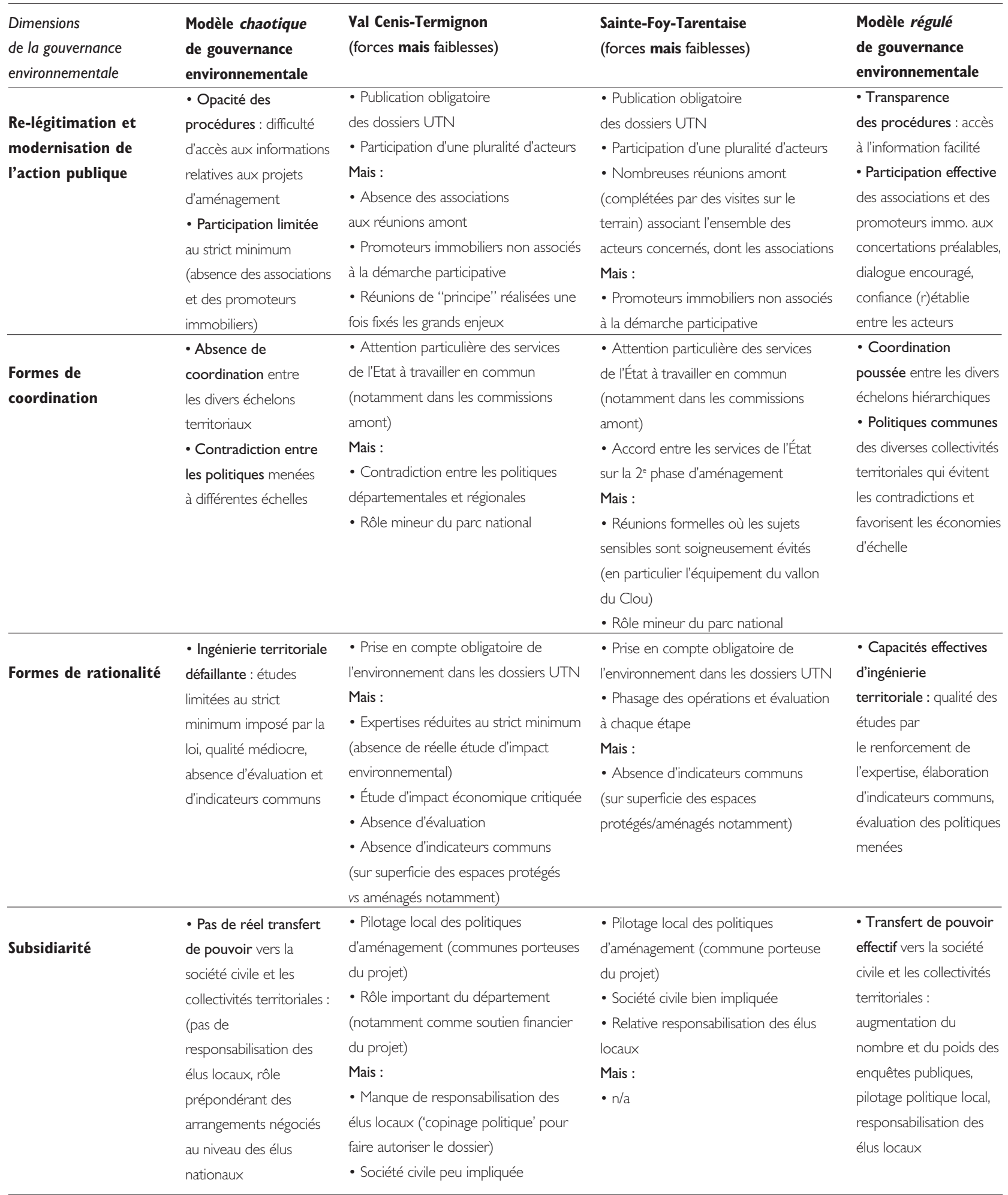


enjeux soulevés par le projet d'aménagement. Au contraire, la perception partagée à Val CenisTermignon était marquée par la frustration et la méfiance. En effet, la plupart des acteurs sont des résidents de longue durée sur les zones d'étude et ont de fait connu différents projets de développement touristique, avec ou sans autorisation d'UTN. Au-delà de leur spécificité, ces deux stations de moyenne montagne soulignent que, si la concertation est au cœur de la gouvernance, elle reste néanmoins une phase sensible. Bien que cristallisant de nombreuses critiques, la concertation reste un temps d'échange entre des groupes d'acteurs aux opinions divergentes, aux ressources variables, aux relations asymétriques et impliqués dans des projets variés. De ce point de vue, une des raisons du succès du dossier d'UTN de Sainte-Foy est liée à l'évitement du problème épineux de l'équipement du vallon du Clou.

Aussi, sans viser un consensus utopique, il s'agit plutôt de souligner les conditions le plus aptes à engendrer une négociation ouverte et respectueuse, ainsi que des règles acceptées par tous en vue de faciliter et de rendre plus efficace ce temps privilégié d'échange. Plus globalement, il convient surtout d'assurer la pérennité de telles conditions de concertation et d'un réel processus de gouvernance environnementale, pour éviter que la décision finale soit perçue et vécue uniquement comme le résultat d'arrangements négociés. Le caractère dynamique des processus de gouvernance environnementale constitue à l'évidence une sorte de variable d'ajustement dans la réalité de la concertation et de son efficacité, dans une perspective de développement durable.

\section{QUELLE ÉVOLUTION DU RÔLE DES SERVICES D'ÉTAT DANS L'AMÉNAGEMENT DES STATIONS ALPINES ?}

En introduisant deux échelles géographiques auxquelles le dossier peut être déposé, le décret de 2006 a redéfini les rôles respectifs des acteurs impliqués dans les UTN. Nos observations confirment que le rôle des services de l'État évolue en termes de ciblage, d'expertise et d'échelle géographique d'aménagement.

En premier lieu, des raisons budgétaires conduisent à une restriction de personnel dans les services de l'État, avec un impact direct sur leur capacité à agir sur une diversité de questions. Les services de l'État tendent à se focaliser à nouveau sur leurs missions centrales. Par exemple, un agent de préfecture a expliqué que : "En ce moment, je suis plutôt sur les questions réglementaires, car on a perdu du personnel et ces questions sont essentielles, et donc je suis moins sur la coordination interministérielle". Ces services ont donc tendance à délaisser des actions qui favoriseraient la gouvernance environnementale, par le dialogue entre les acteurs. Pour Sainte-Foy, notamment : "Il ne s'est rien passé sur la commission de suivi de l'UTN de Sainte-Foy et il serait largement temps de la réunir aujourd'hui. Dans les perspectives anciennes, [...] je prendrais l'initiative [...], mais aujourd'hui, c'est compliqué. C'est un problème des services de l'État".

Les services de l'État doivent donc se réorganiser pour assurer leurs missions, comme le note un agent de préfecture: "Il faut réfléchir à l'organisation préfecture sous-préfecture. [...] Moi je pourrais le faire [l'ingénierie territoriale], mais je fais le boulot des gens qui sont partis car c'est du réglementaire. [...] On n'est peut-être pas obligé de faire de l'urbanisme ici [...], on peut se réorganiser pour éviter les doublons".

Traditionnellement, les services de l'État avaient pour habitude de se rencontrer en amont des procédures d'autorisation UTN pour partager leurs avis et arriver à un “dire d'État" sur le projet. Étant donné que les critères UTN, en particulier au niveau du département, sont moins stricts avec le décret de 2006, il est probable que le nombre de dossiers pour des projets UTN va augmenter, réduisant en cela le temps disponible pour la discussion entre services d'État. Un agent de préfecture tend à le confirmer : "On va avoir du boulot car les cri- 
tères ne sont quand même pas très élevés en commission départementale. Ça m'étonnerait qu'on fasse des commissions amont sur tous les dossiers UTN”.

En second lieu, étant donné les exigences croissantes de transparence et d'efficacité dans l'utilisation des fonds publics, l'État est forcé d'améliorer l'évaluation de ses politiques publiques, et donc ses capacités d'expertise et d'ingénierie territoriale. L'enjeu pour les services de l'État est de justifier les décisions, de les rendre plus acceptables par les collectivités territoriales et les divers acteurs, et d'assurer une mise en œuvre et un suivi de qualité. Ainsi, à propos de la Datar, un élu local déclare: "L'intérêt, c'est qu'ils ont une vision globale du territoire. [...] Donc, ils nous amènent, à travers d'autres expériences, une certaine ouverture d'esprit, une capacité au dialogue, et c'est intéressant. Ils étaient pour faire avancer notre projet, en tenant compte de beaucoup de paramètres, donc on était conseillé plus que correctement et ils ont permis de faire bien avancer le projet".

Cependant, cette capacité d'expertise reste assez limitée, en tout cas pour le moment, pour ce qui est des UTN : le dossier comprend plusieurs études d'impact (économique, environnemental), mais il n'y a pas d'outil d'évaluation spécifique. Un autre élu local rappelle ce besoin d'outil : "Ce serait intéressant d'avoir un fichier en direct répertoriant les dossiers UTN, avec quelques critères simples: date d'instruction, enquête publique...". Disposer d'une telle liste permettrait de replacer tout projet UTN dans un contexte plus général de développement régional et fournirait des indicateurs sur le niveau de rentabilité des sites touristiques, encore mal connu aujourd'hui.

Cette capacité d'expertise est également un moyen, pour les services de l'État, de défendre leur position avec plus de poids et d'avoir un impact plus net sur les décisions prises. Les acteurs locaux reconnaissent ainsi aisément que la DDE (direction départementale de l'équipement) et la Deatm (direction des études et de l'aménagement touristique de la montagne, aujourd'hui délégation montagne d'Atout France) ont une grande expertise technique : "Ils ont une vraie compétence technique”. De son côté, l'ex-Diren (direction régionale de l'environnement) est perçue comme cherchant à améliorer sa capacité d'expertise (et la reconnaissance correspondante) en adaptant sa communication (moins d'accent sur les aspects règlementaires, davantage sur les enjeux d'aménagement régional) et en créant des outils d'aide à la décision : "Il faut des connaissances, un argumentaire pour venir adosser notre discours (qu'on maîtrise complètement sur la partie environnementale) aussi sur des aspects plus économiques et sociaux, du développement durable d'une certaine manière, alors on sera capable d'avoir plus de poids, c'est évident".

Les capacités d'expertise sont également utiles dans la mise en œuvre des décisions. Des comités de suivi sont fréquemment mis en place après les autorisations de projet UTN, mais avec des niveaux d'expertise très variables. Un agent de l'ONF explique : "Il faut savoir quels sont les moyens du comité de suivi. [...] J'ose espérer qu'on voudra bien suivre le soin apporté aux travaux et qu'on aura les moyens d'imposer des travaux". Ce que confirme un agent des servi ces de l'État : "Quasi systématiquement, ces projets [UTN] posent des questions environnementales que les arrêtés d'autorisation n'arrivent pas à régler. [...] C'est là qu'on peut arriver à cadrer un peu les choses, dans les arrêtés d'autorisation, fixer les limites à ne pas dépasser". Par conséquent, des capacités d'expertise accrues permettraient aux services de l'État de suivre et d'appliquer plus facilement la décision prise par le préfet.

Enfin, dans un contexte de réduction du personnel, la question de l'échelle géographique d'aménagement devient prégnante. Le décret UTN de 2006 a renforcé cet enjeu en différenciant deux échelles géographiques pour le dépôt des dossiers (massif $v s$. département), rendant plus difficile une coordination globale des projets de développement touristique, ce qui pourrait conduire à déréglementer l'aménagement de la montagne. Un agent 
des services de l'État affirme : "Chaque préfet va bidouiller [l'autorisation UTN] à sa façon, il n'y aura plus de traitement homogène des demandes, plus personne n'aura de vue d'ensemble". L'État pourrait voir son rôle d'arbitre affaibli dans la mesure où, suite au décret de 2006, il a perdu la fonction de centralisation qui favorise une approche globale de l'aménagement. Comment l'équilibre entre développement touristique et protection des ressources naturelles peut-il être assuré sans vision d'ensemble des enjeux régionaux ?

Par conséquent, l'enjeu majeur pour les services d'État est de décider de l'échelle géographique à laquelle mener l'aménagement durable du territoire. Les perceptions du niveau actuel d'aménagement sont diverses. Ainsi, un représentant d'une association de protection de l'environnement affirme: "Il y a nécessité à trouver un équilibre sur la Tarentaise: le reste de la vallée est largement équipé, d'où l'opposition à un projet local face à l'idée d'équilibre, de cohérence sur la région". Alors que, pour un élu local, "le raisonnement, selon lequel le développement en Tarentaise est terminé, est purement égoïste. On a laissé certains se développer à outrance, ils font (trop) de bénéfices et, à côté de ça, on laisserait mourir ceux qui essaient juste de faire vivre au mieux sans faire de bénéfice (comme nous). Cela ne peut pas tenir, je ne suis pas d'accord". Et dans la vallée de la Maurienne: "On a plus de $45 \%$ du territoire qui est touché par des classements de toute nature. C'est bien, mais il faut aussi que d'autres territoires que la Haute-Maurienne donnent, car il faut aussi qu'on vive!"

L'esprit de clocher et le compromis équipement $v s$. protection ont conduit les services d'État à engager une réflexion intense sur la position à adopter. A été rédigé un document sur l'équilibre entre espaces aménagés et espaces protégés pour préciser les enjeux régionaux, prendre du recul et désamorcer les crises locales. L'objectif était également de fournir des éléments au préfet pour argumenter au mieux ses décisions, et ce en intégrant la dimension spatiale. Il deviendrait alors plus difficile pour les élus locaux d'affirmer que leur projet de développement touristique est spécifiquement rejeté sans raison objective. En plaçant les questions localement sensibles dans un contexte élargi, cette approche semble être un moyen, pour les services d'État, de donner un cadre aux politiques d'aménagement à venir et de développer la gouvernance environnementale dans les stations.

\section{CONCLUSION}

L'objectif de cet article était double : 1) caractériser les processus de gouvernance environnementale dans les stations alpines ; 2) décrire l'évolution du rôle des services d'État dans l'aménagement touris- tique alpin. L'approche méthodologique s'est appuyée sur le cadre théorique de la gouvernance environnementale (Theys, 2003) et sur les demandes d'autorisation d'UTN déposées par deux stations de taille moyenne. À l'aide de documents écrits et d'entretiens auprès d'acteurs, la gouvernance environnementale dans les deux stations a été analysée comme intermédiaire : à mi-chemin entre un modèle chaotique et un modèle régulé. De plus, le rôle des services de l'État a été présenté comme en évolution par rapport au ciblage des missions, à l'expertise et à l'échelle géographique d'aménagement.

Ces deux stations de taille moyenne, situées dans la zone périphérique du même parc national, ayant déposé des demandes d'autorisation UTN au cours de la même période, s'avèrent porteuses d'une problématique plus générique quant à l'articulation entre développement touristique et protection de l'environnement. L'analyse des processus de gouvernance environnementale a mis en relief plusieurs critères pour une lecture critique des UTN : la nature des acteurs et de leurs alliances; le suivi dans le temps et dans l'espace de ces dossiers en vue de qualifier l'impact tant économique qu'environnemental ; le rôle des services d'État à différentes échelles en relation avec les collectivités locales, avec des intérêts divers selon les niveaux territoriaux considérés. Au-delà de la procédure même d'UTN, les 
conclusions mettent en avant des éléments plus globaux autour des aménagements touristiques dans ou à proximité d'espaces à haute valeur patrimoniale.

Les dimensions détaillées par Theys se révèlent utiles pour analyser les processus de gouvernance. Mais cette grille manque de perspective dynamique : elle permet de rendre compte d'une situation à un moment donné, en tout cas sur un laps de temps assez court, mais est plus difficile à appliquer lorsque la configuration des acteurs et des enjeux est susceptible d'évoluer assez fortement. Il est également tentant d'utiliser cette grille pour évaluer une politique publique et sa traduction en termes de "bonne" gouvernance. Or, les éléments qui y sont mobilisés n'apportent pas de réponse quant aux critères classiques d'évaluation des politiques, comme la pertinence ou l'efficacité. Bien que ce ne soit pas l'objectif premier de cette grille, il convient de souligner cette limite vu la force des liens entre politique publique et gouvernance environnementale, a fortiori dans un contexte d'espace protégé.

Cette étude souligne que perspective régionale sur le développement des stations et outils d'évaluation sont nécessaires pour l'aménagement touristique des Alpes françaises. En effet, développer une approche régionale du développement touristique désamorcerait les conflits potentiels en replaçant les projets UTN dans un contexte territorial plus large. En plus de montrer l'importance relative des enjeux environnementaux, sociaux et économiques sur un territoire donné, une telle option faciliterait la compréhension des trajectoires de développement (d'une vallée à l'autre) et l'utilisation de l'analyse par scénarios (tendance vs. situations extrêmes à l'horizon de dix à vingt ans) pour illustrer les enjeux distributifs (aménagement comme protection) et encourager la coopération entre acteurs, qu'ils soient publics ou privés.

Par ailleurs, bien que les dossiers UTN incluent des études d'impact variées, un outil spécifique d'évaluation de la politique UTN serait utile. Selon Vlès (2007), des raisons à la fois démocratiques (les élus et la population devraient savoir comment les fonds publics sont utilisés) et techniques (identification des inefficacités et des moyens pour améliorer les politiques existantes et à venir) poussent à l'évaluation des politiques publiques. Les outils d'évaluation sont maintenant bien acceptés par les acteurs, et beaucoup attendent un suivi et une évaluation des programmes. Des exemples d'indicateurs incluent une liste complète et actualisée des projets UTN sur un territoire donné (suivi temporel et spatial) ou des données sur le taux d'occupation pour estimer la rentabilité des sites touristiques. Disposer d'indicateurs clairement définis favoriserait le dialogue entre acteurs sur des enjeux partagés et favoriserait également des décisions plus "réfléchies", en contradiction éventuelle avec des ambitions locales.

À la suite de cette étude, deux axes complémentaires peuvent être esquissés. Tout d'abord, il serait utile d'étudier l'impact du décret UTN de 2006 au cours du temps, y compris pour savoir si les projets UTN autorisés au niveau du département se traduisent par un éparpillement de petits projets dans les Alpes françaises ("mitage UTN"). Par ailleurs, il serait intéressant de vérifier si les conclusions de cette étude, qui sont générales par leur portée, sont validées dans d'autres pays du massif alpin (Autriche ou Suisse), renseignant ainsi les enjeux de l'aménagement touristique à l'échelle des Alpes.

En conclusion, les autorisations UTN ont été utilisées comme indicateurs des processus de gouvernance environnementale et du rôle en évolution des services de l'État. Cette question devrait aussi être replacée dans le contexte de l'évolution des parcs nationaux français. Une loi sur les parcs votée en 2006 a créé une situation différenciée entre cœur et aire d'adhésion : une protection stricte est maintenue dans le premier, mais soumise à l'adoption d'une charte entre le parc et les communes voisines dans la deuxième. Par conséquent, la protection de l'environnement est plus difficile à assurer dans la zone périphérique. Ainsi, l'adoption de chartes entre parcs et communes partenaires pourrait ouvrir de nou- 
velles perspectives de gouvernance environnementale des stations de sports d'hiver situées à proximité des espaces protégés. Du fait de leur nature, plus permanente que celle des projets UTN, les chartes peuvent avoir un effet de levier pour la gouvernance environnementale des stations, mais le nécessaire processus de négociation peut intensifier les tensions et devenir un enjeu fondamental pour les acteurs concernés.

( I) En particulier, les associations mettent souvent en exergue les exemples des concurrents autrichiens ou suisses germanophones qui développent préférentiellement les mobilités douces par le bas et non les liaisons de stations par le haut (Marcelpoil et al., 2008).

(2) Cette contribution a bénéficié de l'apport du mémoire d'Emeline Hatt (2007). Elle a également bénéficié du soutien financier de la Direction de la nature et des paysages du ministère de l'Écologie et du Développement durable.

(3) Les résultats des UTN ont été généralement jugés comme décevants en raison du taux élevé d'approbation des projets d'aménagement (Merlin, 200I).

(4) Propositions des chercheurs validées par le comité de pilotage du projet, les stations retenues n'étant pas membres de ce comité.

\section{RÉFÉRENCES BIBLIOGRAPHIQUES}

AFIT (Agence française d'ingénierie touristique) (avec Cofremca et New Deal), Carnet de route de la montagne. De l'écoute des clients à l'action marketing Afit, 2003.

Sylvain AllemAND, "Gouvernance : Le pouvoir partagé", Sciences humaines, I0I, 2000.

[http://www.scienceshumaines.com/articleprint2.php?|g=fr\&id_article=9|]

Danielle ArnaUd, La Neige empoisonnée, Alain Moreau, 1975.

ASADAC (Agence savoyarde d'aménagement de développement et d'aide aux collectivités), Dossier d'UTN de Sainte-Foy-Tarentaise, commune de Sainte-Foy, 2004.

Christophe Beaurain, "Gouvernance environnementale locale et comportements économiques. Un nouvel éclairage sur l'articulation du temps et de l'espace", Développement durable et territoires, dossier 2 , 2004. [http://developpementdurable.revues.org/I I I 0]

Pietro Beritelli, Thomas Bieger, Christian LaesSer, "Destination governance: using corporate governance theories as foundation for effective destination management", Journal of Travel Research, 46 (I), 2007.

Christophe Clivaz, Yves Hausser, Jacques Michelet, "Tourism monitoring system based on the concept of carrying capacity. The case of the regional natural park Pfyn-Finges", dans Tuija SIEVÄNEN, Joel ERKKONEN, Jukka JokIMÄKI, Jarkko SAARINEN, Seija TUULENTIE et Eija VIRTANen (dir.), Policies, methods and tools for visitor management. Proceedings of the second international conference on monitoring and management of visitor flows in recreational and protected areas

(Rovaniemi, Finland), 16-20 juin 2004.

Georges Cumin, “Les stations intégrées”, Urbanisme, I 16, 1970.

DCSA, Kalysteo, COMĖte, SIVC, Dossier d'UTN du massif de la Turra, Sivom de Val Cenis, 2005.

Arvid FlAgESTAD et Christine A. Hope, "Strategic success in winter sports destinations: A sustainable value creation perspective", Tourism Management, 22 (5), 2001.

Mickaël Frottier, "UTN : Un mode d'emploi suffisant ?", Montagne Leaders, 20I, 2007.

Françoise GerbauX, Emmanuelle MarCelpoll, "Governance of mountain resorts in France: The nature of the public-private partnership", Revue de géographie alpine, 94(I), 2006. 
Emeline HATT, La Gouvernance environnementale dans les stations de sports d'hiver. Le cas de la procédure UTN, mémoire non publié, université de Pau et des Pays de l'Adour, 2007.

Patrick Le GALÈs, "Du gouvernement des villes à la gouvernance urbaine", Revue française de sciences politiques, 155, 1995.

Emmanuelle MarcelpoIL, Hugues François, Les Stations de moyenne montagne, audition devant le Conseil national de la montagne, 2010.

Emmanuelle Marcelpoil, Christophe Clivaz, Hugues François, "Les stations de sports d'hiver face à la pression immobilière. Quel rôle pour la gouvernance locale?", communication au symposium Innovation et loisirs sportifs de nature (Le Pradel), 19-21 novembre 2008.

Isabelle MAUZ, Gens, cornes et crocs. Relations hommes-animaux et conceptions du monde, en Vanoise, au moment de l'arrivée des loups, thèse de doctorat (sciences de l'environnement), Engref, 2002.

Pierre MerLIN, Tourisme et aménagement touristique. Des objectifs inconciliables?, La Documentation française, 2001.

Harald PECHLANER, "Alpine destination management and marketing in Italy. Transregional cooperation as a factor of competition", Turistica, 8 (2/3), 1999.

Marcel Pérés, "Les unités touristiques nouvelles. Est-ce la montagne qui y gagne?", Tourisme et droit, 89, 2007.

Christophe PérY, La procédure UTN en Haute-Savoie. Les relations entre les services de l'État. La DDE, le SEATM et les communes, mémoire, IEP Grenoble, 1995.

Georges PIALAT, "Urbaniser la montagne ?", Urbanisme, I I 6, 1970. Jorge RIVERA, Peter DE LEON, "Is greener whiter? Voluntary environmental performance of western ski areas", The Policy Studies Journal, 32(3), 2004.

Bo Svensson, Sara Nordin, Arvid Flagestad, "A governance perspective on destination development. Exploring partnerships, clusters and innovation systems", Tourism Review, 60 (2), 2005.

Jacques THEYs, "La gouvernance, entre innovation et impuissance. Le cas de l'environnement", Développement durable et territoires, 2003

[http://developpementdurable.revues.org/document I 523.html]

Vincent VLĖs, Politiques publiques d'aménagement touristique. Objectifs, méthodes, effets, Presses universitaires de Bordeaux, 2006.

Vincent VLĖs, Évaluation de l'expérimentation des schémas d'aménagement et de développement durables de vallée, projet de programme de recherche, 2007.

Robert K. YIN, Case Study Research : Design and methods (4e éd.), Sage, 2009. 